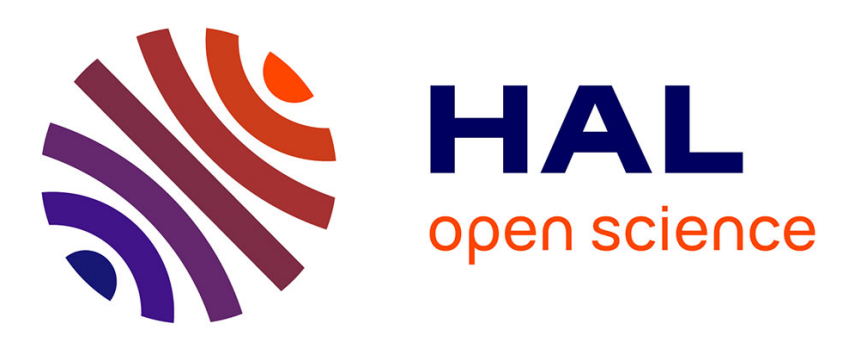

\title{
Hydrodynamics of acoustic phonon-molecular reorientation coupling in plastic crystals; vh scattering \\ E. Courtens
}

\section{To cite this version:}

E. Courtens. Hydrodynamics of acoustic phonon-molecular reorientation coupling in plastic crystals; vh scattering. Journal de Physique Lettres, 1976, 37 (1), pp.21-24. 10.1051/jphyslet:0197600370102100 . jpa-00231222

HAL Id: jpa-00231222

https://hal.science/jpa-00231222

Submitted on 1 Jan 1976

HAL is a multi-disciplinary open access archive for the deposit and dissemination of scientific research documents, whether they are published or not. The documents may come from teaching and research institutions in France or abroad, or from public or private research centers.
L'archive ouverte pluridisciplinaire HAL, est destinée au dépôt et à la diffusion de documents scientifiques de niveau recherche, publiés ou non, émanant des établissements d'enseignement et de recherche français ou étrangers, des laboratoires publics ou privés. 


\title{
HYDRODYNAMICS OF ACOUSTIC PHONON-MOLECULAR REORIENTATION COUPLING IN PLASTIC CRYSTALS; VH SCATTERING
}

\author{
E. COURTENS
}

\author{
IBM Zurich Research Laboratory, 8803 Rüschlikon, Switzerland
}

(Reçu le 8 octobre 1975, révisé le 10 novembre 1975, accepté le 10 novembre 1975)

\begin{abstract}
Résumé. - On développe un modèle qui décrit le couplage entre la réorientation de molécules anisotropes et les modes acoustiques dans un cristal plastique. Le modèle est appliqué au calcul de spectres de diffusion dépolarisée VH pour les cristaux plastiques cubiques. Il prédit deux modes de réorientation suramortis, et l'apparition d'une raie de cisaillement ayant une forme de résonance, en parfait accord avec l'expérience.
\end{abstract}

\begin{abstract}
A model is developed to describe the coupling between the reorientation of anisotropic molecules and acoustic modes in a plastic crystal. The model is applied to the calculation of depolarized VH light-scattering spectra of cubic plastic crystals. It predicts two distinct overdamped reorientation-like modes and the appearance of shear phonon-like modes with resonance lineshapes, in agreement with experimental observation.
\end{abstract}

The statistical reorientation of optically anisotropic molecules gives rise to a depolarized Rayleigh wing in light-scattering spectra, as observed both in liquids [1] and in plastic crystals [2]. From the molecularrotation viewpoint the latter behave very much as liquids, while translationally they are crystalline solids [3]. In liquids there also exists a dynamical coupling between reorientational and translational motion (a static coupling is forbidden) [4] which leads to characteristic light-scattering lineshapes [5]. Their exact interpretation has retained considerable attention [4]. The present letter considers the corresponding situation in plastic crystals. Though their threshold for plastic deformation is small, plastic crystals do sustain static stresses able to lower their symmetry thereby causing molecular reorientation. Hence static correlation between the anisotropic polarizability density and microscopic strains is allowed. This coupling has recently been observed directly for the first time in light-scattering measurements reported separately [6], [7]. The present model gives spectra in full agreement with experiments.

The orientation of molecule a at lattice position $\mathbf{R}_{\mathrm{a}}$ is described by the traceless part $\overleftrightarrow{\alpha}^{a}$ of its optical polarizability tensor (Lorentz-Lorenz corrections will not appear explicitly). Summing over all molecules in volume $V$ one obtains the anisotropic polarizability density

$$
\overleftrightarrow{\mathbf{A}}^{\mathbf{q}}=\sum_{\mathrm{a}} \overleftrightarrow{\boldsymbol{\alpha}}^{\mathrm{a}} \exp \left(-i \mathbf{q} \cdot \mathbf{R}_{\mathrm{a}}\right)
$$

The hydrodynamic anisotropy field is

$$
\overleftrightarrow{\alpha}(\mathbf{r}) \equiv \frac{1}{\mathcal{N}} \sum_{\mathbf{q}} \overleftrightarrow{\mathbf{A}}^{\mathbf{q}} \exp (i \mathbf{q} \cdot \mathbf{r}),
$$

where the sum extends over the $\mathcal{N}$ modes of the first Brillouin zone centered at $\mathbf{q}=\mathbf{0}$ (assuming one molecule per primitive cell, the extension being straightforward). The strain field $\overleftrightarrow{\varepsilon}(\mathbf{r})$ is defined in terms of local displacements $\mathbf{u}$ of the molecular centers by

$$
\varepsilon_{i j}(\mathbf{r}) \equiv\left(\partial u_{i} / \partial x_{j}+\partial u_{j} / \partial x_{i}\right) / 2 .
$$

The Lagrangian density for the coupled system is written

$$
\begin{aligned}
\mathcal{L}=\mathscr{F}-\mathcal{V}=\frac{1}{2} \rho \dot{\mathbf{u}} \cdot \dot{\mathbf{u}}-\frac{1}{2} \overleftrightarrow{\boldsymbol{\varepsilon}} \cdot \overleftrightarrow{C} \cdot \overleftrightarrow{\boldsymbol{\varepsilon}}- \\
-\frac{1}{2} \overleftrightarrow{\boldsymbol{\alpha}} \cdot \overleftrightarrow{\mathbf{B}} \cdot \overleftrightarrow{\alpha}-\overleftrightarrow{\alpha} \cdot \overleftrightarrow{\mathbf{D}} \cdot \overleftrightarrow{\boldsymbol{\varepsilon}} .
\end{aligned}
$$

The first term is the kinetic-energy density which does not include a reorientational contribution as the corresponding modes are usually overdamped. The second term is the elastic potential, and the third term the orientational potential produced by the center of mass interactions and the orientational interactions, respectively. The orientational potential is directly related to the high-frequency Kerr effect [8]. The last term in (2) represents coupling between anisotropy density and strain as explained above. It should be noted that $\overleftrightarrow{\mathbf{C}}$ is the high-frequency elastic tensor. At low frequencies the elastic tensor is dressed by mole- 
cular reorientations. Its value is obtained by minimization of $\vartheta$ with respect to $\overleftrightarrow{\alpha}$ at constant $\overleftrightarrow{\boldsymbol{\varepsilon}}$ and is

$$
\overleftrightarrow{\mathbf{C}}^{\text {eff }}=\overleftrightarrow{\mathbf{C}}-\overleftrightarrow{\mathbf{D}} \cdot \overleftrightarrow{\mathbf{B}}^{-1} \cdot \overleftrightarrow{\mathbf{D}}
$$

For cubic crystals of point group higher than $\mathrm{T}$ there are three elastic constants in $\overrightarrow{\mathbf{C}}$, two orientational constants in $\overleftrightarrow{\mathbf{B}}$, and two coupling constants in $\overleftrightarrow{\mathbf{D}}$. Indeed, the tracelessness of $\overleftrightarrow{\alpha}$ allows to impose

$$
B_{11}+2 B_{12}=D_{11}+2 D_{12}=0,
$$

using the abbreviated subscript notation. The dressed low-frequency $\mathbf{C}^{\text {eff }}$ is given by

$$
\begin{aligned}
C_{11}^{\text {eff }}+ & 2 C_{12}^{\text {eff }}=C_{11}+2 C_{12}, C_{11}^{\text {eff }}-C_{12}^{\text {eff }}= \\
& =C_{11}-C_{12}-\left(D_{11}-D_{12}\right)^{2} /\left(B_{11}-B_{12}\right),
\end{aligned}
$$

and

$$
C_{44}^{\text {eff }}=C_{44}-D_{44}^{2} / B_{44} .
$$

Static stability demands that $v$ be positive definite, which in the cubic case requires that $C_{11}+2 C_{12}$, $C_{11}-C_{12}, C_{44}, B_{11}-B_{12}, B_{44}, C_{11}^{\text {eff }}-C_{12}^{\text {eff }}$, and $C_{44}^{\text {eff }}$ all be positive.

The presence of frictional forces is accounted for by a dissipative function equal to half the rate of entropy production [9]. Results obtained in this manner are equivalent to those obtained with the more usual hydrodynamic calculation of entropy production following the lines of irreversible hydrodynamics [10]. Each potential term having its associated dissipative part one writes

$$
f=\frac{1}{2} \dot{\vec{\varepsilon}} \cdot \stackrel{\leftrightarrow}{\Gamma}^{\varepsilon} \cdot \dot{\vec{\varepsilon}}+\frac{1}{2} \dot{\vec{\alpha}} \cdot \stackrel{\leftrightarrow}{\Gamma^{\alpha}} \cdot \dot{\vec{\alpha}}+\dot{\vec{\alpha}} \cdot \stackrel{\leftrightarrow}{\Gamma}^{\alpha \varepsilon} \cdot \dot{\vec{\varepsilon}}
$$

where the $\overleftrightarrow{\boldsymbol{\Gamma}}$ 's are transport coefficients having a microscopic expression in terms of time dependent correlation functions [11]. The symmetries of the $\overleftrightarrow{\boldsymbol{\Gamma}}$ 's are identical to those of the potential constants. One also defines a dressed elastic loss $\overleftrightarrow{\boldsymbol{\Gamma}}^{\varepsilon, \text { eff }}$ which is the high-frequency loss as seen below. Dynamical stability requires that $f$ be positive definite, leading to conditions on the $\Gamma^{\prime}$ s similar to those on the potential constants.

Application of Lagrange equations of motion for continuous dissipative systems gives a set of eight coupled eq. [12] :

$$
\begin{gathered}
\rho \ddot{u}_{i}-C_{i j k l} \partial^{2} u_{k} / \partial x_{j} \partial x_{l}-D_{i j k l} \partial \alpha_{k l} / \partial x_{j}= \\
=\Gamma_{i j k l}^{\varepsilon} \partial^{2} \dot{u}_{k} / \partial x_{j} \partial x_{l}+\Gamma_{i j k l}^{\alpha \varepsilon} \partial \dot{\alpha}_{k l} / \partial x_{j}, \\
B_{i j k l} \alpha_{k l}+D_{i j k l} \partial u_{k} / \partial x_{l}= \\
=-\Gamma_{i j k l}^{\alpha} \dot{\alpha}_{k l}-\Gamma_{i j k l}^{\alpha \varepsilon} \partial \dot{u}_{k} / \partial x_{l},
\end{gathered}
$$

where tensor symmetries have been used to replace strains by unsymmetrized gradients. In the absence of coupling ( $\left.\mathbf{D}=\overleftrightarrow{\boldsymbol{\Gamma}}^{\alpha \varepsilon}=0\right)$, (4a) gives the usual acoustic phonons with the $q^{2}$ dependence of their width, and (4b) gives overdamped reorientation modes. With coupling and in the high-frequency limit the lefthand side of $(4 b)$ becomes negligible and the right-hand side gives $\dot{\alpha}$ in terms of $\nabla \dot{u}$. The introduction of this value in (4a) shows that $\overrightarrow{\boldsymbol{\Gamma}}^{\text {eeff }}$ is the high-frequency loss. Eq. (4) are space Fourier transformed using (1) and a similar relation between $\mathbf{u}(\mathbf{r})$ and $\mathbf{U}^{\mathbf{q}}$, and then Laplace transformed with the notation

$$
\overleftrightarrow{\mathcal{A}}^{\mathbf{q}}(s)=\int_{0}^{\infty} \mathrm{e}^{-s t} \overleftrightarrow{\mathbf{A}^{\mathbf{q}}}(t) \mathrm{d} t,
$$

and similarly for $\boldsymbol{U}^{\mathbf{q}}(s)$. For a general direction $\mathbf{q}$ an $8 \times 8$ system results. However for $q$ in the (110) plane one identifies in the cubic case, to which the remaining discussion is restricted, a subset of three coupled equations between

$$
\mathcal{U} \equiv\left(\mathcal{U}_{1}-\mathcal{U}_{2}\right) / \sqrt{2}, \quad \mathcal{A}_{1} \equiv\left(\mathfrak{A}_{11}-\mathfrak{A}_{22}\right) / \sqrt{2},
$$

and

$$
\mathcal{A}_{2} \equiv \sqrt{2}\left(\mathcal{A}_{13}-\mathcal{A}_{23}\right) .
$$

This geometry has the advantage that only one pure shear mode is active in VH scattering. In that case $\mathcal{A}_{1}$ and $\mathcal{A}_{2}$ are also the only components needed to calculate the spectrum.

The depolarized spectrum produced by molecular motion (excluding deformation of electronic shells and anisotropic local fields) is given by [13]

$$
\begin{aligned}
I(\mathbf{q}, \omega) & \propto V I_{0} \operatorname{Re} \int \mathrm{d} t \mathrm{e}^{i \omega t} \frac{1}{2 \mathcal{N}}\left\langle\sum_{\mathbf{a}, \mathbf{b}} \alpha_{i j}^{\mathrm{a}}(t) \alpha_{k l}^{\mathrm{b}}(0) \times\right. \\
& \left.\times \exp \left\{-i \mathbf{q} \cdot\left[\mathbf{r}_{\mathrm{a}}(t)-\mathbf{r}_{\mathrm{b}}(0)\right]\right\}\right\rangle n_{i}^{0} n_{j}^{s} n_{k}^{0} n_{l}^{s},
\end{aligned}
$$

where $\omega$ is the frequency shift, $I_{0}$ is the incident intensity, and $V$ is the scattering volume containing $\mathcal{N}$ lattice points. The sum extends over all pairs. The position of molecule a is $\mathbf{r}_{\mathrm{a}}=\mathbf{R}_{\mathrm{a}}+\mathbf{u}_{\mathrm{a}}$. The polarization vectors of the incident and scattered fields are $\mathbf{n}^{0}$ and $\mathbf{n}^{\mathbf{s}}$, respectively. The angular bracket indicates ensemble averaging. The correlation range of $\overleftrightarrow{\alpha}^{a}$ and $\overleftrightarrow{\alpha}^{b}$ being finite, the bracketed part reduces to $\left\langle A_{i j}^{\mathbf{q}}(t) A_{k l}^{\mathbf{q}}(0)\right\rangle+\cdots$. The further terms are smaller by a factor of the order of $|\mathbf{q . u}|^{2}$ and are negligible. These additional terms could not be neglected if $\overleftrightarrow{\alpha}^{a}$ and $\overleftrightarrow{\alpha}^{b}$ had long-range correlation, as in the case of polarized scattering, or for orientationally ordered anisotropic molecules. These terms contribute then to the usual Pockel's piezooptic coefficients. For the VH scattering geometry of figure 1 , and with

$$
\mathbf{n}^{\mathbf{s}}=[1,1,2 m] /\left(2+4 m^{2}\right)^{1 / 2},
$$

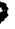
one obtains from (5) and the definitions above :

$$
\begin{aligned}
I(\mathbf{q}, \omega) & \propto\left[V I_{0} / \mathcal{N}\left(4+8 m^{2}\right)\right] \times \\
& \times \operatorname{Re}\left\langle\mathcal{A}_{1}^{\mathbf{q}}(s=-i \omega) A_{1}^{-\mathbf{q}}(t=0)\right. \\
& \left.+m \mathcal{A}_{1}^{\mathbf{q}} A_{2}^{-\mathbf{q}}+m \mathcal{A}_{2}^{\mathbf{q}} A_{1}^{-\mathbf{q}}+m^{2} \mathcal{A}_{2}^{\mathbf{q}} A_{2}^{-\mathbf{q}}\right\rangle,
\end{aligned}
$$


where the arguments are explicitly shown in the first bracketed term.

Solving the Laplace transformed equations, the dynamical correlation functions entering (6) are expressed in terms of static correlation functions such as $\left\langle A_{1}^{q} A_{1}^{-\mathbf{q}}\right\rangle$. The latter are calculated from equipartition using the Hamiltonian

$$
\mathscr{H}=\int \mathrm{d} V(\mathfrak{F}+\mathcal{Y}) .
$$

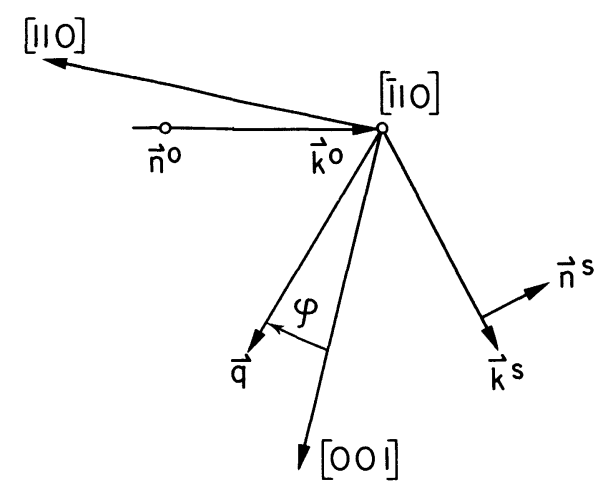

FIG. 1. - The scattering geometry : $\mathbf{n}_{0}$ and $[\overline{1} 10]$ are perpendicular to the plane of the paper, $\mathbf{k}^{0}$ and $\mathbf{k}^{s}$ are the incident and scattered wavevectors, respectively, and $\mathbf{q}$ is the momentum exchange.

The results are simplified by defining new constants

$$
\begin{gathered}
B_{1} \equiv B_{11}-B_{12}, \quad B_{2} \equiv B_{44}, \\
D_{1} \equiv\left(D_{11}-D_{12}\right) / \sqrt{2}, \quad D_{2} \equiv D_{44}, \\
C \equiv\left[C_{44}+\frac{1}{2}\left(C_{11}-C_{12}-2 C_{44}\right) \sin ^{2} \varphi\right],
\end{gathered}
$$

where

$$
\mathbf{q}=q[\sin \varphi, \sin \varphi, \sqrt{2} \cos \varphi] / \sqrt{2} .
$$

The undressed phonon velocity is written $v=\sqrt{C / \rho}$. New dissipative constants $\Gamma_{1}^{\alpha}, \Gamma_{2}^{\alpha}, \Gamma_{1}^{\alpha \varepsilon}, \Gamma_{2}^{\alpha \varepsilon}$, and $\Gamma^{\varepsilon}$ are defined similarly. Finally, the dynamical equations are simplified by using complex constants of the type $\mathfrak{B}_{1}=B_{1}+s \Gamma_{1}^{\alpha}$, and similarly for $\mathfrak{B}_{2}, \mathfrak{D}_{1}, \mathfrak{D}_{2}$ and $\mathrm{C}$. The poles of the response function, which are the eigenfrequencies of the new hybrid modes, are given by the quartic

$$
\begin{aligned}
\Re_{1} \Re_{2}\left(\rho s^{2}+\mathcal{C} q^{2}\right)-q^{2} & \cos ^{2} \varphi \Re_{1} D_{2}^{2}- \\
& -q^{2} \sin ^{2} \varphi \Re_{2} \mathfrak{D}_{1}^{2}=0 .
\end{aligned}
$$

These poles are written $s_{j}=z_{j}+\delta_{j}(j=1$ to 4$)$, where the $z_{j}$ 's are the values in the absence of coupling. One identifies two reorientational modes (assumed non-degenerate)

$$
z_{j}=-B_{j} / \Gamma_{j}^{\alpha}(j=1,2)
$$

and the complex conjugated phonon modes, which for small phonon damping are $z_{3,4} \cong \pm i v q-\Gamma^{\varepsilon} q^{2} / 2 \rho$. Lowest order in the couplings one finds then

$$
\delta_{1}=\left[q^{2} \sin ^{2} \varphi D_{1}^{2} / \rho \Gamma_{1}^{\alpha}\left(s^{2}+v^{2} q^{2}\right)\right]_{s=z_{1}},
$$

with a similar expression for $\delta_{2}$ obtained by interchange of the subscripts 1 and 2 and of $\sin \varphi$ and $\cos \varphi$, whereas for the phonon

$$
\begin{aligned}
\delta_{3,4}=\left[( q ^ { 2 } / 2 \rho s ) \left(D_{1}^{2} \sin ^{2} \varphi / \mathfrak{B}_{1}+\right.\right. \\
\left.\left.+\mathscr{D}_{2}^{2} \cos ^{2} \varphi / \mathscr{B}_{2}\right)\right]_{s= \pm i v q} .
\end{aligned}
$$

The depolarized spectrum is the sum of three components $I_{1}+I_{2}+I_{P}$. The first two are Rayleigh wings corresponding to $s_{1}$ and $s_{2}$. Using (6) one finds to same order in the couplings :

$$
\begin{aligned}
I_{1}(\mathbf{q}, \omega) & \propto \frac{I_{0} \pi \mathcal{N}}{\beta \Gamma_{1}^{\alpha}\left|s_{1}\right|} \frac{2}{1+2 m^{2}} L\left(\omega, s_{1}\right) \times \\
& \times\left\{1+\frac{q^{2} \sin \varphi \mathfrak{D}_{1}}{\rho\left(s^{2}+v^{2} q^{2}\right)} \cdot\left[2 \sin \varphi \frac{v^{2} q^{2} \Gamma_{1}^{\alpha \varepsilon}-s D_{1}}{\Gamma_{1}^{\alpha}\left(s^{2}+v^{2} q^{2}\right)}\right.\right. \\
+ & \left.\left.\frac{2 m \cos \varphi D_{2}+m^{2} \sin \varphi D_{1}}{\mathfrak{B}_{2}}\right]\right\}_{s=z_{1}}, \quad(9 a)
\end{aligned}
$$

where $\beta=1 / k T$, and $L\left(\omega, s_{1}\right) \equiv\left|s_{1}\right| / \pi\left(\omega^{2}+s_{1}^{2}\right)$ is the normalized Lorentzian profile. The component $I_{2}(\mathbf{q}, \omega)$ is obtained from $I_{1}(\mathbf{q}, \omega)$ by the same interchange as above, and replacing $m$ by $1 / m$ within curly brackets only, after which the whole expression is multiplied by $\mathrm{m}^{2}$. The third component corresponds to $s_{3,4}$. It consists of the Stokes and anti-Stokes parts of the shear phonon. In lowest order

$$
\begin{aligned}
I_{P}(\mathbf{q}, \omega) & \propto\left[I_{0} \pi \mathcal{N} / \beta C\left(1+2 m^{2}\right)\right] \times \\
& \times \operatorname{Re}\left\{\left[P\left(\omega, s_{3}\right)+P\left(-\omega, s_{3}\right)\right]\right. \\
& \left.\times\left[\sin \varphi D_{1} / \mathscr{B}_{1}+m \cos \varphi \mathbb{D}_{2} / \mathfrak{B}_{2}\right]^{2}\right\}_{s=i v q},
\end{aligned}
$$

where

$$
\begin{aligned}
& P(\omega,-\Gamma+i \Omega)= \\
& \quad=[\Gamma+i(\Omega-\omega)] / \pi\left[\Gamma^{2}+(\Omega-\omega)^{2}\right]
\end{aligned}
$$

$I_{P}$ is purely Lorentzian in the limits of very small or very small or very large $q$. For intermediate $q$ it has a resonance or Breit-Wigner lineshape [14]. Moreover, if the dissipative part of the coupling is small, the line disappears at high $q$.

A measurement as a function of crystal orientation determines unambiguously all parameters of the theory including the relative sign of all four coupling constants. An excellent agreement is obtained between theory and experiment in succinonitrile [6]. The results can also be compared to the low-frequency elastic constants $\overrightarrow{\boldsymbol{C}}^{\text {eff }}$ known from ultrasonics, and to the high-frequency optical Kerr-effect susceptibility $\overleftrightarrow{\chi}^{N L}$ which is a $\mathbf{q}=t=0$ measurement of the correlation function within angular brackets in eq. (5). From equipartition one obtains

$$
B_{1}=N^{2} L_{P}^{2} L_{0} / 2\left(\chi_{11}^{N L}-\chi_{12}^{N L}\right),
$$

where $N$ is the number of molecules per unit volume 
and $L_{P}, L_{0}$ are Lorentz-Lorenz local field factors [8]. The ratio $B_{1} / B_{2}$ is related to the optical Kerr-effect anisotropy $\zeta$ by $B_{1} / B_{2}=2-4 \zeta$.

Finally one should note that (5) implies that the anisotropic part of the Pockels piezooptic effect $\overleftrightarrow{\mathbf{p}}$ arises entirely from the coupling considered here. Without coupling $p_{44}=p_{11}-p_{12}-2 p_{44}=0$ and the pure shear mode does not scatter light. This in fact is approximately true for highly symmetric systems such as the rare-gas solids [15]. It thus seems legitimate to account for the shear-phonon scattering strength observed in plastic crystals entirely on the basis of the present-coupling. In cases where the observed phonons have a frequency much above the reorientational frequency their strength would come from the dissipative terms $\overleftrightarrow{\Gamma}^{\alpha \varepsilon}$, whereas in the opposite limit it would come from the static term $\overleftrightarrow{\mathbf{D}}$. Detailed comparison of theory and experiment would be needed to distinguish these contributions from piezooptic effects of other origins.

Acknowledgements. - The author expresses his appreciation to Prof. N. Gershon, J. Feder, and H. Thomas for a number of fruitful discussions.

\section{References}

[1] Starunov, V. S., Tiganov, E. V. and Fabelinkskil, I. L., JETP Lett. 4 (1966) 176.

[2] Boyer, L., Vacher, R., Cecchi, L., Adam, M. and Bergé, P., Phys. Rev. Lett. 26 (1971) 1435.

[3] Timmermans, J., J. Phys. \& Chem. Solids 18 (1961) 1.

[4] Gershon, N. D. and OpPenheIM, I., Physica 64 (1973) 247.

[5] Stegeman, G. I. A. and Stoicheff, B. P., Phys. Rev. Lett. 21 (1968) 202 and Phys. Rev. 7A (1973) 1160.

[6] Bischofberger, T. and Courtens, E., to be published.

[7] An indirect observation of a similar type of coupling has been made in solid $\mathrm{H}_{2}$ where it leads to an anomalous strength for neutron scattering from librons [STEIN, H., STILLER, H. and Stockmeyer, R., J. Chem. Phys. 57 (1972) 1726].

[8] Bischofberger, T. and Courtens, E., Phys. Rev. Lett. 32 (1974) 163 and Courtens, E., Phys. Rev. 10A (1974) 967.

[9] Landau, L. D. and Lifshitz, E. M., in Statistical Physics (Pergamon Press, London, England) 1958, Chapter XII.

[10] De Groot, S. and Mazur, P., in Non-Equilibrium Thermodynamics (North-Holland, Amsterdam, Holland) 1969, and Gershon N. D. and Courtens E., to be published.
[11] Balescu, R., in Equilibrium and Nonequilibrium Statistical Mechanics (Wiley, N. Y., USA) 1975, Chapters 12 and 21.

[12] The approach is justified by extending to continuous systems considerations made by MACHLUP, S. and ONSAGER, L., Phys. Rev. 91 (1953) 1512. For a variable $\eta$ the Lagrangian equation is

$$
\begin{aligned}
\frac{\partial}{\partial t}(\partial \mathcal{L} / \partial \dot{\eta})+\sum_{k} \frac{\partial}{\partial x_{k}}\left[\partial \mathcal{L} / \partial\left(\partial \eta / \partial x_{k}\right)\right]-\partial \mathcal{L} / \partial \eta & = \\
= & -\partial f / \partial \dot{\eta}+\sum_{k} \frac{\partial}{\partial x_{k}}\left[\partial f / \partial\left(\partial \dot{\eta} / \partial x_{k}\right)\right] .
\end{aligned}
$$

[13] Ben Reuven, A. and Gershon, N. D., J. Chem. Phys. 51 (1969) 893.

[14] Breit, G. and Wigner, E. P., Phys. Rev. 49 (1936) 519; Fano, U., Phys. Rev. 124 (1961) 1866.

[15] Gornall, W. S. and Stolcheff, B. P., Phys. Rev. 4B (1971) 4518. 